\title{
Infected Cavitating Pulmonary Infarction
}

\author{
Srinivas Rajagopala MD DM, Uma Devaraj DNB, and George D'Souza MD DNB
}

\section{Introduction}

The differential diagnosis of pulmonary cavitation is broad. The most common causes include infection and malignancy. Pulmonary thromboembolism is a common problem worldwide, with an estimated 650,000 cases in the United States annually. Pulmonary embolism does not usually lead to cavitation, given the dual blood supply of the lung; however, cavitation may complicate $4-7 \%$ of all pulmonary infarctions. We present an elderly diabetic woman with coronary artery disease who presented with symptoms suggestive of a lung abscess. Re-evaluation because of persistent unexplained breathlessness and radiologic non-response led to the diagnosis of an infected submassive pulmonary embolism and infarction as the cause of cavitation. We discuss the common causes of pulmonary cavitation, with special emphasis on embolism, and the radiologic appearance and management of pulmonary cavitation.

\section{Case Summary}

A 60-year-old woman presented with fever, productive cough, right-side pleuritic chest pain, and breathlessness of one month's duration. Her illness started with lowgrade fever, breathlessness, and pleuritic pain, noticed after waking up one morning. She received oral antibiotics empirically, with no response. A week later, purulent cough with postural variation and streaky hemoptysis developed. Breathlessness worsened to limit daily activities. Her medical history was notable for poorly controlled diabetes with retinopathy and nephropathy. She had undergone coronary artery bypass grafting 4 years prior to her current symp-

Srinivas Rajagopala MD DM, Uma Devaraj DNB, and George D’Souza MD DNB are affiliated with the Division of Chest Diseases, Department of Medicine, St John's Medical College Hospital, Bangalore, India.

The authors have disclosed no conflicts of interest.

Correspondence: Srinivas Rajagopala MD DM, Division of Chest Diseases, Department of Medicine, St John's Medical College Hospital, Sarjapur Road, Bangalore India 560034. E-mail: visitsrinivasan@ gmail. com.

DOI: $10.4187 /$ respcare. 00828 toms, and had been asymptomatic during that period. She was a homemaker and denied any smoking, alcohol, or substance abuse. She was evaluated in another hospital after the onset of fever and cough. Chest radiograph and high-resolution computed tomography (CT) (Fig. 1) showed a right-side lung abscess. Sputum cultures revealed Klebsiella pneumoniae sensitive to piperacillin-tazobactam, but Zeil-Neelson-stain smears for acid-fast bacilli were negative. She received 2 weeks of parenteral antibiotics, with reduction of sputum, but she continued to have severe pleuritic pain and breathlessness at rest. She was referred to our center for evaluation. Her medications at the time of evaluation included aspirin, clopidogrel, atorvastatin, enalapril, metoprolol, insulin, piperacillin-tazobactam, diclofenac, and pantoprazole

On evaluation she was afebrile, alert, and normotensive, with a respiratory rate of 26 breaths/min and a heart rate of 110 beats/min. There was no evidence of pedal edema or generalized lymphadenopathy. Auscultation revealed coarse crackles with bronchial breathing in the right mammary area. Laboratory investigations showed microcytic hypochromic anemia $(9.3 \mathrm{~g} / \mathrm{dL}$, mean corpuscular volume $72 \mathrm{fL}$ ), leukocytosis, and normal platelet count. Renal function tests were remarkable for elevated serum creatinine (1.4 mg/dL) and 24-hour urine protein $\left(600 \mathrm{mg} / \mathrm{m}^{2} / \mathrm{d}\right)$. Urine microscopy, serum electrolytes, and liver function tests were normal. Fasting serum glucose was $160 \mathrm{mg} / \mathrm{dL}$ and hemoglobin A1c was 7.5\%. Fundoscopy confirmed non-proliferative diabetic retinopathy bilaterally. Q waves in inferior leads, right axis deviation, and poor $\mathrm{R}$ wave progression in frontal leads were seen in the electrocardiogram. Transthoracic echocardiography showed no evidence of infective endocarditis, normal left-ventricular function, mild tricuspid regurgitation, and pulmonary artery hypertension (right ventricular systolic pressure $36 \mathrm{~mm} \mathrm{Hg}$ ). Human immunodeficiency virus enzymelinked immunosorbent assay was non-reactive, and the Mantoux test was negative $(6 \mathrm{~mm})$. Arterial blood analysis revealed $\mathrm{P}_{\mathrm{aO}_{2}} / \mathrm{F}_{\mathrm{IO}_{2}} 325 \mathrm{~mm} \mathrm{Hg}, \mathrm{P}_{\mathrm{aCO}_{2}} 30 \mathrm{~mm} \mathrm{Hg}$, pH 7. 48, and $\mathrm{HCO}_{3} 23 \mathrm{mEq} / \mathrm{L}$. Repeat chest radiograph (see Fig. 1) showed a large thin-walled irregular right-mid-zone lung abscess with no air/fluid level, ipsilateral effusion, and sternotomy sutures. Sputum culture was positive for En- 


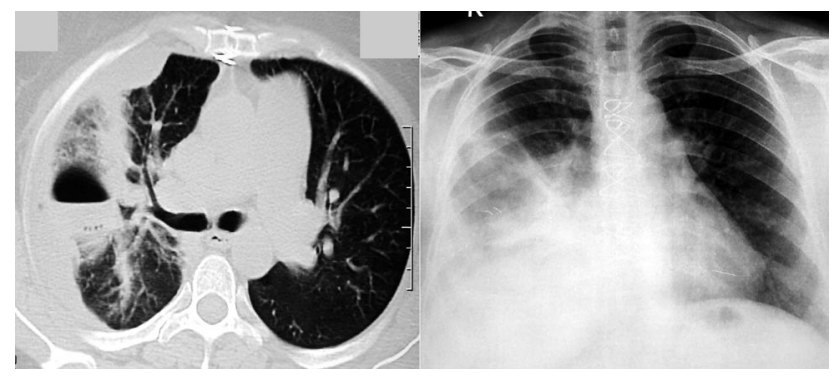

Fig. 1. Left: Computed tomogram 1 week after onset of symptoms shows a right-middle lobe lung abscess with an air/fluid level and small ipsilateral pleural effusion. Right: Chest radiograph 2 weeks after the onset of symptoms shows a thin-walled irregular rightmid-zone lung abscess without no air/fluid level.

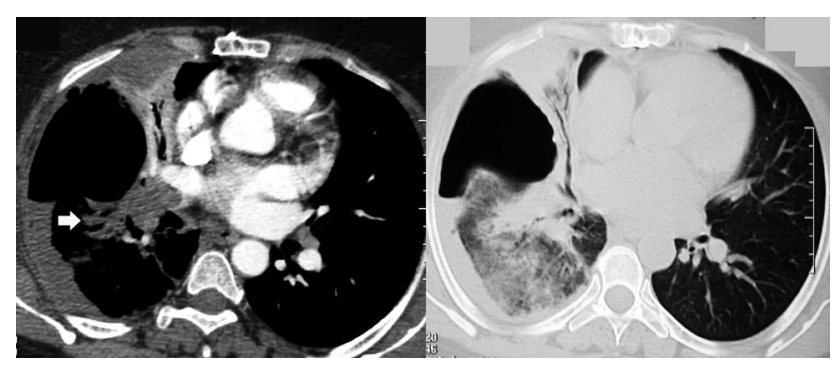

Fig. 2. Contrast-enhanced computed tomogram shows a large hypo-attenuating clot in the entire right main pulmonary artery and extending into the right upper and descending pulmonary artery. Left: A small pleural effusion is also evident. Right: High-resolution lung slice shows a thin-walled irregular lung abscess with scalloped inner margins and surrounding consolidation with air bronchograms.

terobacter species sensitive to meropenem, but negative for acid-fast bacilli, Mycobacterium tuberculosis (via BACTEC, BD, Franklin Lakes, New Jersey), and fungi (via Sabouraud dextrose agar). Anti-nuclear antibodies and anti-neutrophil cytoplasmic antibodies were negative (via immunofluorescence).

Because of persistent breathlessness and pleuritic pain, contrast-enhanced CT was performed after adequate hydration and parenteral $\mathrm{N}$-acetylcysteine, to rule out pulmonary embolism and malignancy. The contrast-enhanced CT showed a large lung abscess in the right middle lobe, with scalloped margins, peripheral consolidation, a small pleural effusion, and a large hypoechoic clot in the right main pulmonary artery (Fig. 2, left). CT pulmonary angiogram showed complete occlusion of the right main pulmonary artery, with no contrast passage in the entire right side (Fig. 3). A diagnosis of infected cavitating pulmonary infarction was made.

\section{Discussion}

In this patient, the presence of severe unexplained breathlessness at the onset of illness, the characteristic radiologic

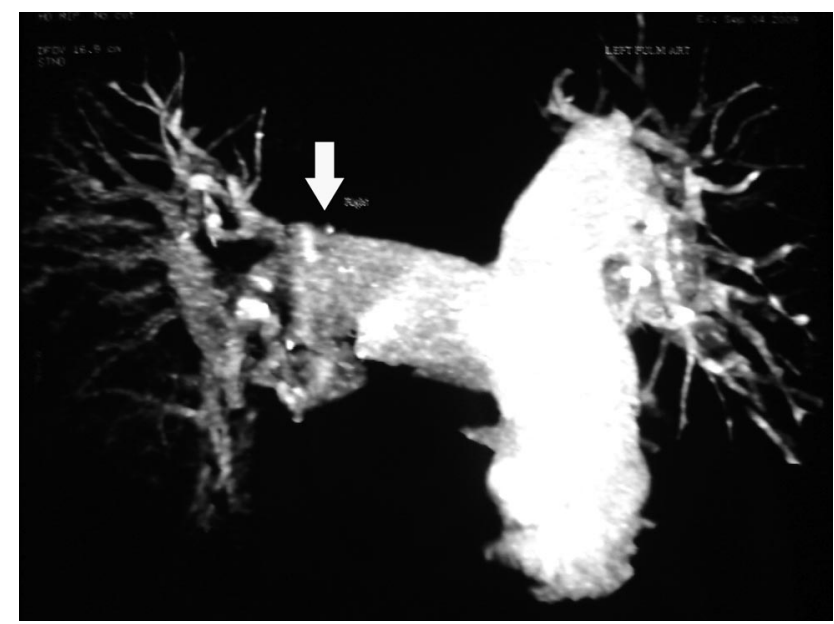

Fig. 3. Three-dimensional reconstruction of the computed tomogram pulmonary angiogram shows lack of contrast passage in the entire right side.

Table 1. Differential Diagnosis of Pulmonary Cavitation

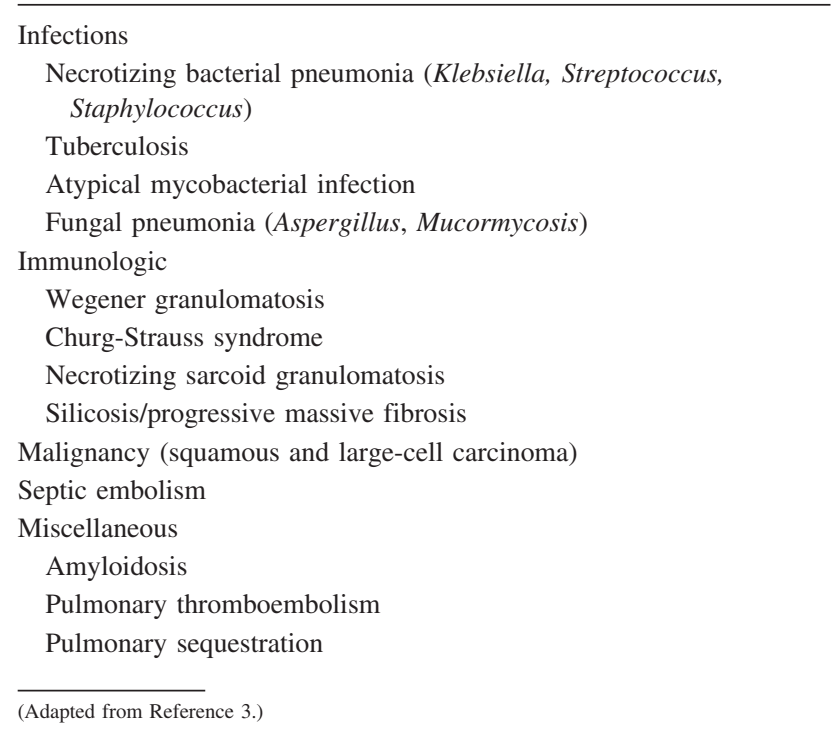

features, the echocardiography findings, and the extensive anatomical embolism favor infected cavitating pulmonary embolism as the cause of cavitation, and make in-situ thrombosis complicating lung abscess unlikely. The differential diagnosis for pulmonary cavitation is broad (Table 1). Pulmonary infarction is a well recognized cause of cavitation $^{1}$ and accounted for about $1 \%$ of all non-tuberculous cavities in the pre-CT era. ${ }^{2}$ Pulmonary infarction occurs in only $10 \%$ of pulmonary embolism, given the dual nature of pulmonary blood flow. ${ }^{3}$ Aseptic liquefaction and cavitation may occur in bland infarctions larger than $4 \mathrm{~cm}$ in diameter. ${ }^{4}$ Cavitation complicates about $4-7 \%$ of pulmonary infarctions. ${ }^{5}$ 
Cavitation is a delayed complication of pulmonary infarction, mostly in elderly patients with comorbid conditions, usually congestive cardiac failure. ${ }^{5}$ These cavities are usually single and occur predominantly on the right side. While Libby et al ${ }^{5}$ reported no lobar predominance, preponderance in the apical or posterior segment of the upper lobe or the apical segment of the lower lobe has been reported. ${ }^{4}$ Prominent fever and purulent sputum indicates super-added infection. ${ }^{1,5}$ Radiograph features of constant cavity size, irregular cavity outline, and no fluid level may suggest a bland (ie, not infected) cavity. ${ }^{1}$ Computed tomogram features of scalloped inner margins and cross-cavity band shadows have also been reported to favor cavitating pulmonary infarction. ${ }^{1}$ Cavitation occurs earlier in an infected embolism (mean $18 \mathrm{~d}$, range 6-40 d) than in a bland infarct (mean $28 \mathrm{~d}$, range $7-120 \mathrm{~d}$ ). ${ }^{5}$ Complications such as pneumothorax, empyema, and bronchopleural fistula have been reported in bland infarctions ${ }^{6}$ but are more common in infected infarctions. Gram-negative bacteria are the most common organisms causing superadded infection. Earlier series reported high mortality (56\%): higher in infected infarctions $(73 \%)$ than in bland infarctions $(41 \%)$. No recent series have examined mortality in this subset of patients, but in the past decade, mortality is probably much lower, given the improved recognition, earlier diagnosis, improved imaging of and therapies and supportive care for massive pulmonary embolism.

We managed our patient with subcutaneous heparin and oral warfarin, targeting an international normalized ratio of 2. Parenteral meropenem was administered for 2 weeks. Thrombolysis was not considered because of the long duration of symptoms and the absence of hypotension, hypoxemia, and substantial right-ventricular dysfunction. Peripheral smear, gynecologic evaluation, mammography, colonoscopy and ultrasound abdomen (paraneoplastic work up) were normal. She improved symptomatically without any recurrence of hemoptysis and was asymptomatic at 2-month follow-up. She completed one year of anticoagulation, and warfarin was stopped for 3 months, with no recurrence.

\section{Teaching Points}

- Cavitation occurs in less than $1 \%$ of all pulmonary embolisms, because of the dual nature of pulmonary blood flow.

- Computed tomographic features of scalloped inner margins and cross-cavity band shadows suggest a cavitating pulmonary infarction.

- Prominent fever and purulent sputum indicate an infected cavitating pulmonary infarction.

- Management is with anticoagulation and appropriate antibiotics.

\section{REFERENCES}

1. Scharf J, Nahir AM, Munk J, Lichtig C. Aseptic cavitation in pulmonary infarction. Chest 1971;59(4):456-458.

2. Laforet EG, Laforet MT. Nontuberculous cavitary disease of the lungs. Dis Chest 1957;31(6):665-679.

3. Fishman AP, Elias JA, Fishman JA, Grippi MA, Senior RM, Pack AI, editors. Fishman's pulmonary diseases and disorders, 4th edition. New York: McGraw-Hill; 2008.

4. Wilson AG, Joseph AE, Butland RJ. The radiology of aseptic cavitation in pulmonary infarction. Clin Radiol 1986;37(4):327-333.

5. Libby LS, King TE, LaForce FM, Schwarz MI. Pulmonary cavitation following pulmonary infarction. Medicine (Baltimore) 1985; 64(5):342-348.

6. Hall FM, Salzman EW, Ellis BI, Kurland GS. Pneumothorax complicating aseptic cavitating pulmonary infarction. Chest 1977;72(2): 232-234. 